$10-2000$

\title{
Fluid Mechanic Assessment of the Total Cavopulmonary Connection using Magnetic Resonance Phase Velocity Mapping and Digital Particle Image Velocimetry
}

\author{
Ann E. Ensley \\ Georgia Technical University \\ Agnès Ramuzat \\ Georgia Technical Univeristy \\ Timothy M. Healy \\ GoprgiäTechnical University

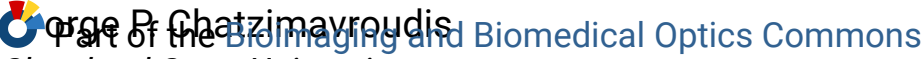 \\ Gleveland State University dow work benefit you? Let us know! \\ Earblisibar's Statement

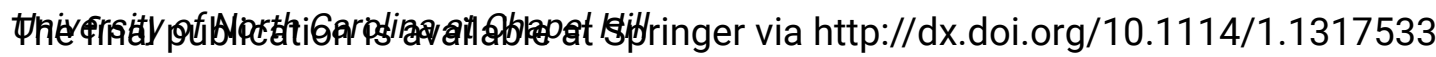

\section{Oeiginal Dathors}

Ensley AE, Ramuzat A, Healy TM, Chatzimavroudis GP, Lucas C, Sharma S, Pettigrew R, Yoganathan AP. Fluid Mechanic Assessment of the Total Cavopulmonary Connection using Magnetic Resonance Phase Velocity Mapping and Digital Particle Image Velocimetry. Ann Biomed Eng. 2000;28:1172-1183.

\section{Repository Citation}

Ensley, Ann E.; Ramuzat, Agnès; Healy, Timothy M.; Chatzimavroudis, George P.; Lucas, Carol; Sharma, Shiva; Pettigrew, Roderic; and Yoganathan, Ajit P., "Fluid Mechanic Assessment of the Total Cavopulmonary Connection using Magnetic Resonance Phase Velocity Mapping and Digital Particle Image Velocimetry" (2000). Chemical \& Biomedical Engineering Faculty Publications. 101.

https://engagedscholarship.csuohio.edu/encbe_facpub/101

This Article is brought to you for free and open access by the Chemical \& Biomedical Engineering Department at EngagedScholarship@CSU. It has been accepted for inclusion in Chemical \& Biomedical Engineering Faculty Publications by an authorized administrator of EngagedScholarship@CSU. For more information, please contact library.es@csuohio.edu. 


\section{Authors}

Ann E. Ensley, Agnès Ramuzat, Timothy M. Healy, George P. Chatzimavroudis, Carol Lucas, Shiva Sharma, Roderic Pettigrew, and Ajit P. Yoganathan 


\title{
Fluid Mechanic Assessment of the Total Cavopulmonary Connection using Magnetic Resonance Phase Velocity Mapping and Digital Particle Image Velocimetry
}

\author{
Ann E. Ensley, ${ }^{1}$ Agnès Ramuzat, ${ }^{1}$ Timothy M. Healy, ${ }^{1}$ George P. Chatzimavroudis, ${ }^{2}$ \\ Carol Lucas, ${ }^{3}$ Shiva Sharma, ${ }^{4}$ Roderic Pettigrew, ${ }^{5}$ and AJit P. Yoganathan ${ }^{5}$ \\ ${ }^{1}$ Georgia Tech/Emory Department of Biomedical Engineering, Atlanta, GA, ${ }^{2}$ Department of Chemical Engineering, Cleveland State \\ University, Cleveland, OH, ${ }^{3}$ Biomedical Engineering Department, University of North Carolina, Chapel Hill, NC, ${ }^{4} \mathrm{Children}$ 's \\ Heart Center, Emory University School of Medicine, Atlanta, GA, and ${ }^{5}$ Frederik Philips Magnetic Resonance Research Center, \\ Emory University School of Medicine, Atlanta, GA
}

\begin{abstract}
The total cavopulmonary connection (TCPC) is currently the most promising modification of the Fontan surgical repair for single ventricle congenital heart disease. The TCPC involves a surgical connection of the superior and inferior vena cavae directly to the left and right pulmonary arteries, bypassing the right heart. In the univentricular system, the ventricle experiences a workload which may be reduced by optimizing the cavae-to-pulmonary anastomosis. The hypothesis of this study was that the energetic efficiency of the connection is a consequence of the fluid dynamics which develop as a function of connection geometry. Magnetic resonance phase velocity mapping (MRPVM) and digital particle image velocimetry (DPIV) were used to evaluate the flow patterns in vitro in three prototype glass models of the TCPC: flared zero offset, flared $14 \mathrm{~mm}$ offset, and straight $21 \mathrm{~mm}$ offset. The flow field velocity along the symmetry plane of each model was chosen to elucidate the fluid mechanics of the connection as a function of the connection geometry and pulmonary artery flow split. The steady flow experiments were conducted at a physiologic cardiac output (4 L/min) over three left/right pulmonary flow splits $(70 / 30,50 / 50$, and 30/70) while keeping the superior/ inferior vena cavae flow ratio constant at 40/60. MRPVM, a noninvasive clinical technique for measuring flow field velocities, was compared to DPIV, an established in vitro fluid mechanic technique. A comparison between the results from both techniques showed agreement of large scale flow features, despite some discrepancies in the detailed flow fields. The absence of caval offset in the flared zero offset model resulted in significant caval flow collision at the connection site. In contrast, offsetting the cavae reduced the flow interaction and caused a vortex-like low velocity region between the caval inlets as well as flow disturbance in the pulmonary artery with the least total flow. A positive correlation was also found between the direct caval flow collision and increased power losses. MRPVM was able to elucidate these important fluid flow features, which may be important in future modifications in TCPC surgical designs. Using MRPVM, two- and threedirectional velocity fields in the TCPC could be quantified. Because of this, MRPVM has the potential to provide accurate
\end{abstract}

velocity information clinically and, thus, to become the in vivo tool for TCPC patient physiological/functional assessment.

Keywords-Fontan, Single ventricle, Hypoplastic left heart.

\section{INTRODUCTION}

In children with single ventricle congenital heart disease, surgical intervention is often the only survival option. In 1971 Fontan and Baudet ${ }^{6}$ introduced an innovative surgical approach for treatment of tricuspid atresia. Over the years, this original "Fontan" procedure has been modified as a result of many numerical, experimental, and clinical investigations ${ }^{9,10,12,17}$ and currently is used as a palliative procedure for many complex anomalies. The most promising modification is the total cavopulmonary connection (TCPC). The TCPC involves surgical connection of the superior and inferior vena cava (SVC and IVC, respectively) to the unbranched right pulmonary artery (RPA). This palliative surgical procedure leads to a separation between oxygenated and deoxygenated blood, which is critical for effective oxygen transport to the body. As a result of this modified circulation, the single ventricle experiences an increased workload, pumping blood to both the systemic circulation and to the lungs. This workload can be reduced by altering the cavae-to-pulmonary anastomosis to minimize the fluid mechanical energy losses at the connection. Previous research has demonstrated that improving the anastomosis geometry can reduce the energy loss within the connection, ${ }^{4,8,17}$ and therefore lead to more successful surgical outcomes. The hypothesis of this study is that the fluid mechanics of the TCPC, which develop as a result of the connection geometry and pulmonary flow split, are an important indicator of the efficiency and thus the physiologic success of the connection. 
The long-term clinical goals are to improve the efficiency of the TCPC and to develop a technique for noninvasive patient assessment. But, first a methodology for evaluating potential connection designs in vitro, which can also be implemented in vivo, is needed. In vitro, the relative efficiency of a given surgical design can be quantified from a control volume analysis using direct measurement of pressure and volumetric flow. However, clinically, pressure measurements are highly invasive and potentially unreliable due to limited sensitivity and unreliable catheter placement in the complex flow fields observed in the TCPC. In contrast, measurements of two- and three-directional velocity is possible, noninvasively, using magnetic resonance phase velocity mapping.

In this study, a comparison of two velocity measurement techniques was performed. Magnetic resonance phase velocity mapping (MRPVM) and digital particle image velocimetry (DPIV) were used to evaluate the flow patterns in three prototype models of the TCPC. DPIV, an established fluid mechanic technique, has been used in limited biological applications, ${ }^{1,7,11,15}$ but is a powerful technique to instantaneously quantify the twodimensional velocity over a large field of view. Unfortunately, DPIV is limited to in vitro applications. ${ }^{16,19}$ MRPVM can provide measurement of all three spatial components of the velocity vector, not only in vitro, but also in vivo noninvasively. This unique ability of MRPVM may be the key for physiologic in vivo TCPC patient assessment, and in making the correct surgical plan to optimize the cavopulmonary connection. Previous studies have evaluated MRPVM, ${ }^{2,3,5,13,18}$ but this study is the first to compare the results of MRPVM and DPIV in prototype TCPC models, in order to elucidate the fluid mechanics of the cavopulmonary connection. Establishing MRPVM as a tool to evaluate the performance of the TCPC will have invaluable clinical significance. The first aim of this study was to evaluate the potential of MRPVM in assessing physiologic information about the fluid mechanics in the TCPC through a comparison of the flow fields obtained with MRPVM and DPIV. The second aim was to investigate the effects of the connection geometry and pulmonary flow split on the flow patterns and the resulting energy efficiency of the TCPC.

\section{METHODS}

\section{Total Cavopulmonary Connection Models}

Three custom crafted glass models of the TCPC were used to study the effect of the cavopulmonary anastomosis geometry on the local fluid mechanics. These models were designed to look at three surgically relevant anastomosis geometries. Two models incorporated flaring of the connection [Figs. 1(a) and 1(b)], with 0 and $14 \mathrm{~mm}$ (1 caval diameter) offsets between caval centers. The
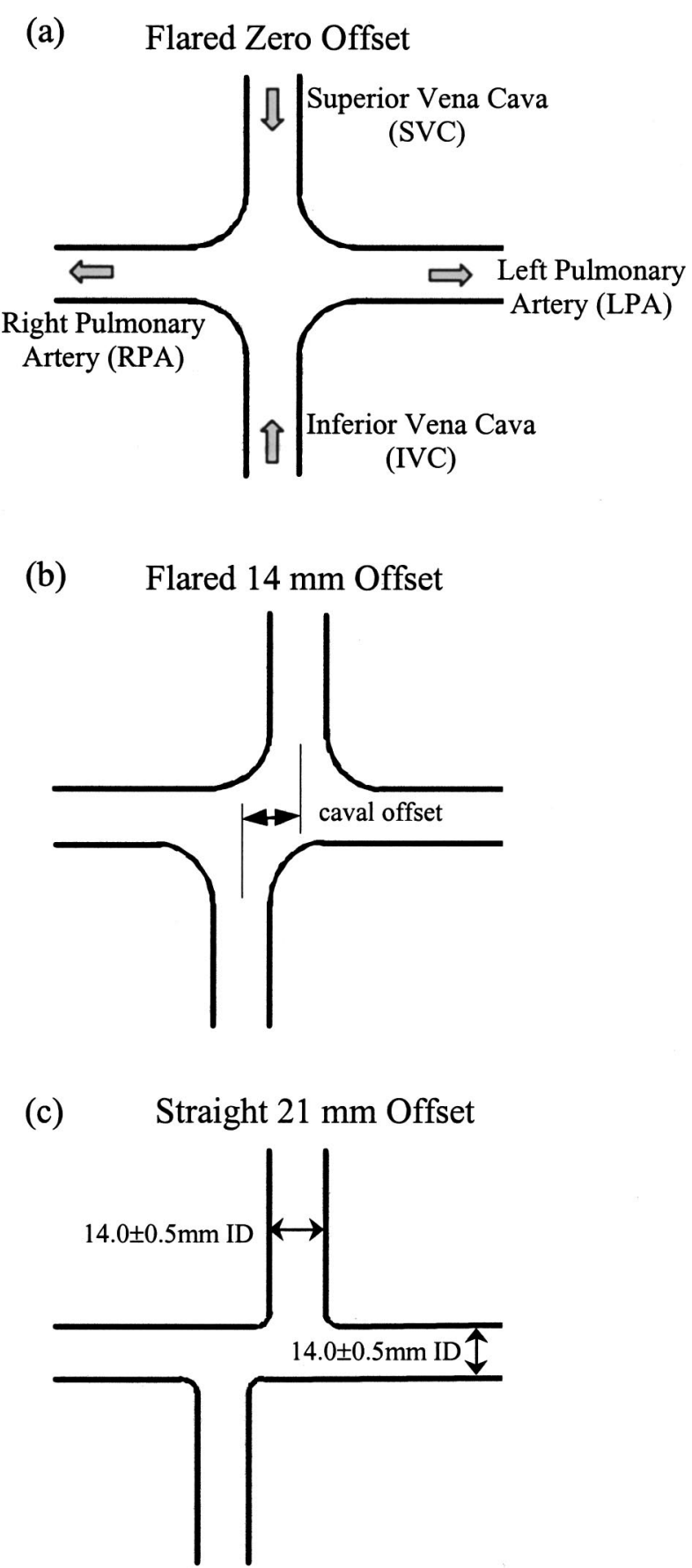

FIGURE 1. Schematic of the three custom crafted glass prototype total cavopulmonary connection (TCPC) models. (a) The flared zero offset model incorporated flaring at the anastomosis site with zero offset between caval centers. (b) The flared $14 \mathrm{~mm}$ (1 caval diameter) offset model incorporated flaring at the anastomosis site with a $14 \mathrm{~mm}$ offset between caval centers. (c) The straight $21 \mathrm{~mm}$ (1.5 caval diameter) offset model was formed with smoothed perpendicular connections between the cavae and pulmonary arteries with a 21 $\mathrm{mm}$ offset between caval centers.

third model [Fig. 1(c)] only incorporated the effect of caval offset, without flaring at the anastomosis site (straight $21 \mathrm{~mm}$ offset or 1.5 caval diameter offset). The 
TABLE 1. Summary of the experimental conditions.

\begin{tabular}{|c|c|c|c|c|c|c|c|}
\hline Model & $\begin{array}{c}\text { Pulmonary } \\
\text { flow split } \\
\text { to RPA }\end{array}$ & $\begin{array}{l}\text { Vessel inner } \\
\text { diameter } \\
(\mathrm{cm})\end{array}$ & $\begin{array}{l}\text { DPIV } \\
\text { working } \\
\text { fluid } \\
\text { viscosity } \\
\left(\mathrm{cm}^{2} / \mathrm{s}\right)\end{array}$ & $\begin{array}{c}\text { DPIV } \\
\text { flow rate } \\
\text { (L/min) }\end{array}$ & $\begin{array}{c}\text { Reynolds } \\
\text { No. }\end{array}$ & $\begin{array}{l}\text { MRPVM } \\
\text { working } \\
\text { fluid } \\
\text { viscosity } \\
\left(\mathrm{cm}^{2} / \mathrm{s}\right)\end{array}$ & $\begin{array}{c}\text { MRPVM } \\
\text { flow rate } \\
\text { (L/min) }\end{array}$ \\
\hline \multirow[t]{2}{*}{ Flared $14 \mathrm{~mm}$ offset } & $70 \%$ of total & \multirow{3}{*}{1.42} & \multirow{3}{*}{0.035} & 2.80 & 1194 & \multirow{3}{*}{0.01006} & 0.80 \\
\hline & $50 \%$ of total & & & 2.00 & 853 & & 0.57 \\
\hline Flared zero offset & $30 \%$ of total & & & 1.20 & 512 & & 0.34 \\
\hline \multirow{3}{*}{ Straight $21 \mathrm{~mm}$ offset } & $70 \%$ of total & \multirow{3}{*}{1.35} & \multirow{3}{*}{0.035} & 2.80 & 1261 & \multirow{3}{*}{0.01006} & 0.80 \\
\hline & $50 \%$ of total & & & 2.00 & 901 & & 0.57 \\
\hline & $30 \%$ of total & & & 1.20 & 540 & & 0.34 \\
\hline
\end{tabular}

flared model cavae to pulmonary artery connections had a radius of curvature of approximately $10 \mathrm{~mm}$. Anatomic MRI data of an eight year old TCPC patient was used to model the internal diameter of the cavae and pulmonary arteries at $14.0 \pm 0.5 \mathrm{~mm}$.

\section{Flow Loop}

A steady flow loop was constructed to accommodate each prototype model for flow field investigation. The flow loop consisted of a series of PVC tubes, and ball valves, which provided resistance to control the desired flow rates. For the DPIV studies, measurement of flow rate was performed via three rotometers (Models 6B0202 and 6B0204, Dakota Instruments, Monsey, NY). One rotometer was used to determine the total flow rate delivered to both venae cavae, a second was used to measure the flow rate in one inlet (IVC), and the third was used to measure the flow rate in one outlet (RPA). In the MRPVM studies, a MRI compatible brass transit time ultrasonic flow probe (Model 24-N in-line, Transonic Systems Inc., Ithaca, NY) was used to measure the outlet flow rate in the RPA.

\section{Experimental Conditions (DPIV and MRPVM)}

The flow conditions for evaluating each TCPC model were representative of the physiologic flow rates. The DPIV experiments were run at a clinically relevant cardiac output (total flow rate) of $4 \mathrm{~L} / \mathrm{min}$. The working fluid was chosen to match the kinematic viscosity of blood in large vessels $(3.5 \pm 0.1 \mathrm{cSt})$ and to match the refractive index of the glass models $(n D=1.4728)$ to prevent optical distortion. The approximate constituent concentration of the fluid was $79 \%$ saturated sodium iodide solution, $20 \%$ glycerin, and $1 \%$ deionized water by volume. The refractive index was measured with a refractometer (Model 2192, Extech Instruments Corporation, Waltham, MA) and the fluid viscosity was measured with a Cannon Fenske routine viscometer, size 100 (Fisher Scientific, Pittsburgh, PA). An acrylic box con- taining the refractive index matched solution was also built around the model to further reduce any errors due to refraction on the curved model edges. The superiorto-inferior venae cavae flow ratio (SVC/IVC) was held constant at 40:60 in all experiments. Three pulmonary flow splits were studied: $70 \%, 50 \%$, and $30 \%$ of the total flow to the RPA. The flow split was adjusted by controlling the pulmonary vessel resistance.

These experiments were designed to allow a direct comparison of the TCPC flow dynamics measured with both MRPVM and DPIV. Because of the operational limitations encountered when using a clinical MRI scanner, water (kinematic viscosity $=1.0 \mathrm{cSt}$ ) was used as the working fluid, instead of the aqueous $\mathrm{NaI} /$ glycerin solution used with DPIV. In order to establish similarity between the MRPVM and DPIV studies, the ratio of inertial to viscous forces was scaled using Reynolds number similarity. Dynamic similarity was important because this study was specifically interested in the fluid mechanics of the cavopulmonary connection as a function of geometry and pulmonary flow split. Similarity was established using the vessel inner diameter, which remained constant, and the average velocity for a given flow rate. Table 1 summarizes the experimental flow conditions.

\section{Digital Particle Image Velocimetry Measurements}

DPIV uses digitally recorded video images to investigate the instantaneous and average velocity flow fields. The current study was performed using a commercial DPIV system (TSI Incorporated, St. Paul, MN). As shown in Fig. 2, it consisted of a PC-controlled synchronizer directly connected to a pulsed laser and to a charge coupled device (CCD) video camera. The symmetry plane of each TCPC model was illuminated with a NdYAG pulsed laser (500 $\mu$ s pulse delay), which was reflected through an articulated arm and both a cylindrical and spherical lens to produce a laser light sheet approximately $1 \mathrm{~mm}$ thick. The fluid was seeded with $10 \mu \mathrm{m}$ 

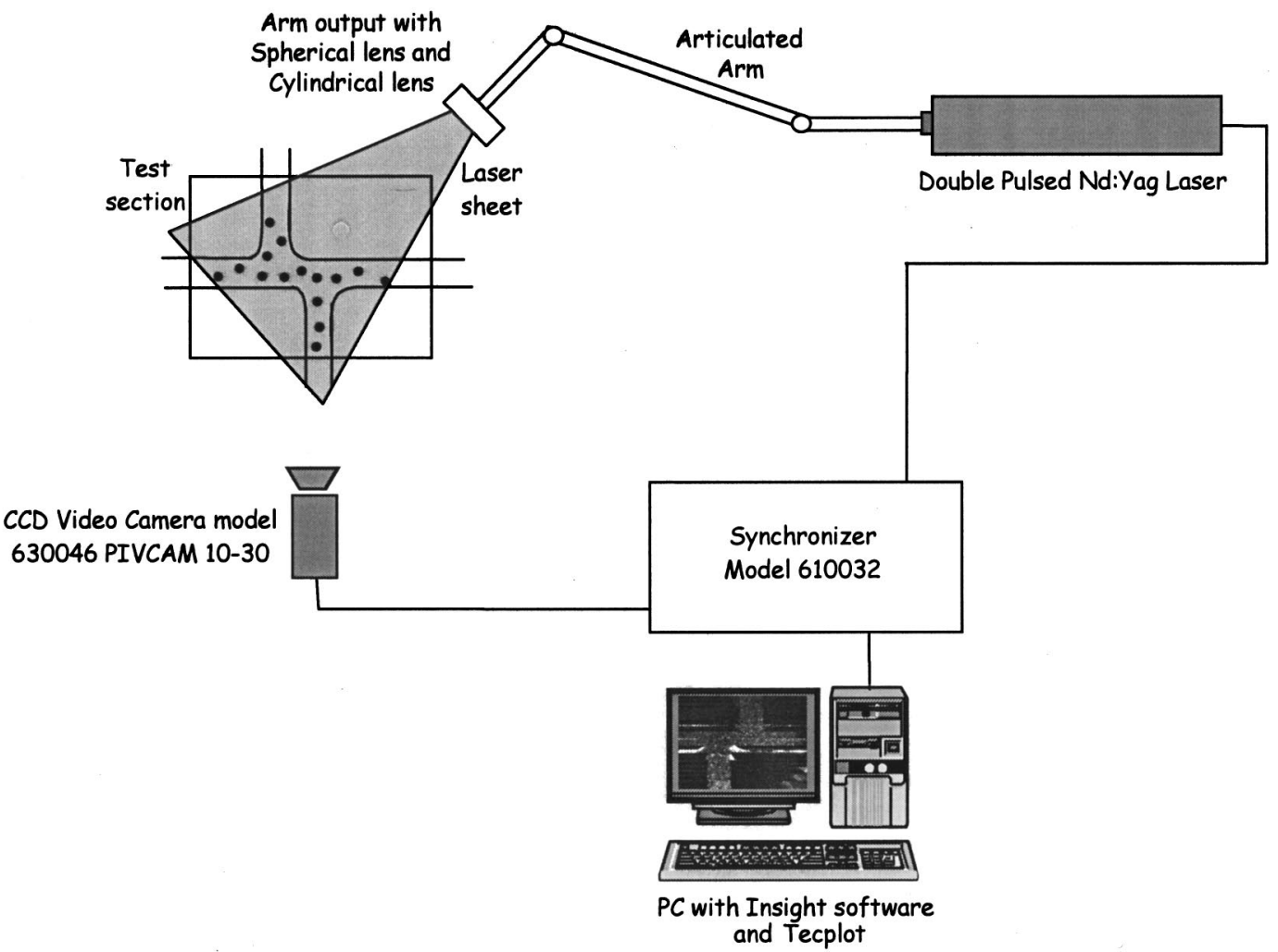

FIGURE 2. Schematic of the DPIV system used in this study. The system was composed of a PC controlled synchronizer interfaced to a CCD video camera and a pulsed mini-YAG laser which illuminated the model test section as shown.

diameter hollow glass spheres (Model 10089, TSI Fluid Mechanics Division, St. Paul, MN) which reflected the laser light. The CCD camera had a resolution of 1008 $\times 1018$ pixels and was focused normal to the illuminated plane. The images were collected using a frame grabber, and stored for off-line analysis. This measurement setup allowed the acquisition of 32 successive raw images, which yielded 16 instantaneous $2 \mathrm{D}$ velocity fields along the model symmetry plane (approximately $0.1 \mathrm{~s}$ apart) after cross correlation. Images were acquired at each flow condition yielding an instantaneous and average 2D velocity field.

\section{Data Analysis-DPIV}

The images were processed using Insight ${ }^{\mathrm{TM}}$ software (TSI Incorporated, St. Paul, MN) which yielded 2D velocity vector maps over the region of interest. The processing of the acquired images was done with a twoframe cross correlation using a Gaussian peak search algorithm and interrogation windows of $64 \times 64$ pixels. The two scaler components of velocity $\left(v_{x}\right.$ and $\left.v_{y}\right)$ were combined to obtain the velocity vector field. A postprocessing vector field validation program (EditVec, TSI Inc., St. Paul, MN) was used to eliminate spurious vectors as a result of lost pairs due to out-of-plane motion or low seeding areas which cause a low correlation signal strength. The program used a global range filter to remove any erroneous values. Then, the mean of the neighboring $3 \times 3$ vectors was used to replace that value. Less than $5 \%$ of the vectors were considered spurious based on the limits of the global range filter.

Visualization of the data was done using a commercial software program (Tecplot version 7.5, Amtec Engineering, Bellevue, WA). Calibration of the velocity from displacement/time to $\mathrm{m} / \mathrm{s}$ was performed in Tecplot, based on the model tube diameter.

\section{Magnetic Resonance Phase Velocity Mapping}

The MRPVM images used in this study were acquired using a $1.5 \mathrm{~T}$ whole-body MRI scanner (Gyroscan ACS, Philips Medical Systems, Best, The Netherlands). Each TCPC model was mounted in a water-filled plastic container to ensure adequate signal, centered in the bore of the magnet, and connected to the flow loop via polyurethane tubing. Coronal, sagittal, and axial spin echo scout images were initially acquired to localize the TCPC models inside the scanner and to ensure that each model was aligned along the traditional superior-inferior, right-left, and anterior-posterior scanner directions. These images served as a reference from which a $4 \mathrm{~mm}$ 
TABLE 2. Imaging parameters for the gradient-echo velocity encoding acquisitions.

\begin{tabular}{ll}
\hline \multicolumn{1}{c}{ Pulse sequence } & Gradient-echo-FFE \\
\hline Number of slices & 1 \\
Slice thickness $(\mathrm{mm})$ & 4 \\
Field of view $(\mathrm{mm} \times \mathrm{mm})$ & $200 \times 200$ \\
Flip angle $(\mathrm{deg})$ & 35 \\
Repetition time $(\mathrm{ms})$ & 30 \\
Echo time $(\mathrm{ms})$ & $6-9$ \\
Number of signals averaged & 4 \\
Matrix size (pixels) & $256 \times 256$ \\
Velocity encoding value $(\mathrm{cm} / \mathrm{s})$ & $10-20$ \\
\hline
\end{tabular}

slice (centered along the symmetry plane of each model) was selected for flow quantification. Velocity data were acquired using a FFE gradient-echo pulse sequence to encode velocity along the superior-inferior, right-left, and anterior-posterior directions. The imaging parameters for the flow quantification sequence are summarized in Table 2.

\section{Data Analysis-MRPVM}

The MRPVM images were transferred from the scanner to a Silicon Graphics Onyx mainframe (Silicon Graphics, Mountain View, CA) for storage. Image masking, data translation, and visualization were then performed on a Windows-based PC (Toshiba America Information Systems, Inc., Irvine, CA). Transform (Fortner Research, LLC, Sterling, VA) was used to convert the phase data to velocity, select the region of interest (ROI),

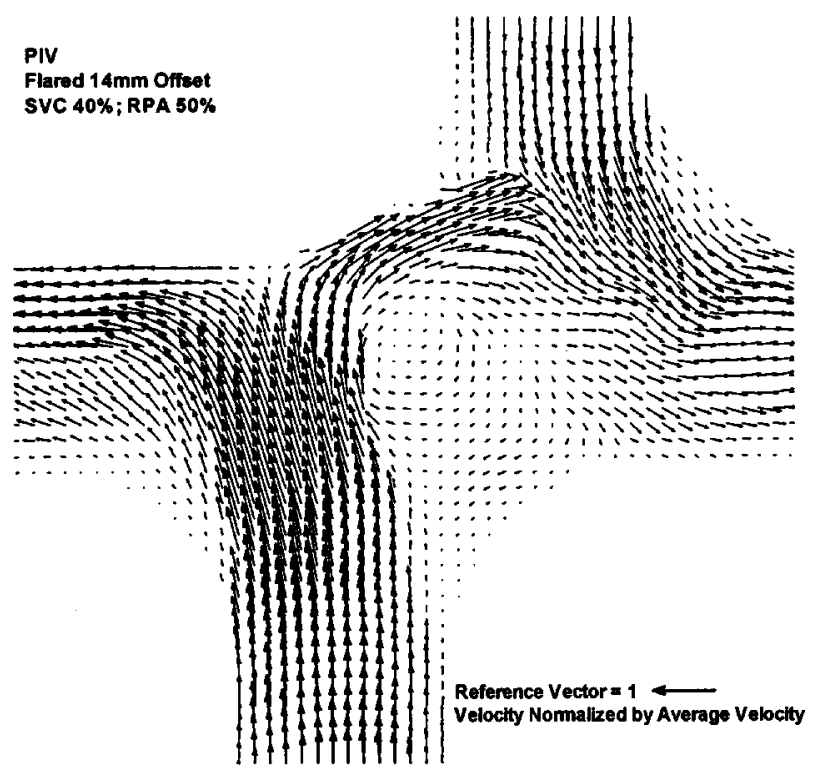

FIGURE 3. Digital particle image velocimetry vector plot of flow in the flared $14 \mathrm{~mm}$ (1 caval diameter) offset TCPC model. The SVC:IVC flow ratio was $40: 60$ with $50 \%$ of the total flow to the RPA.

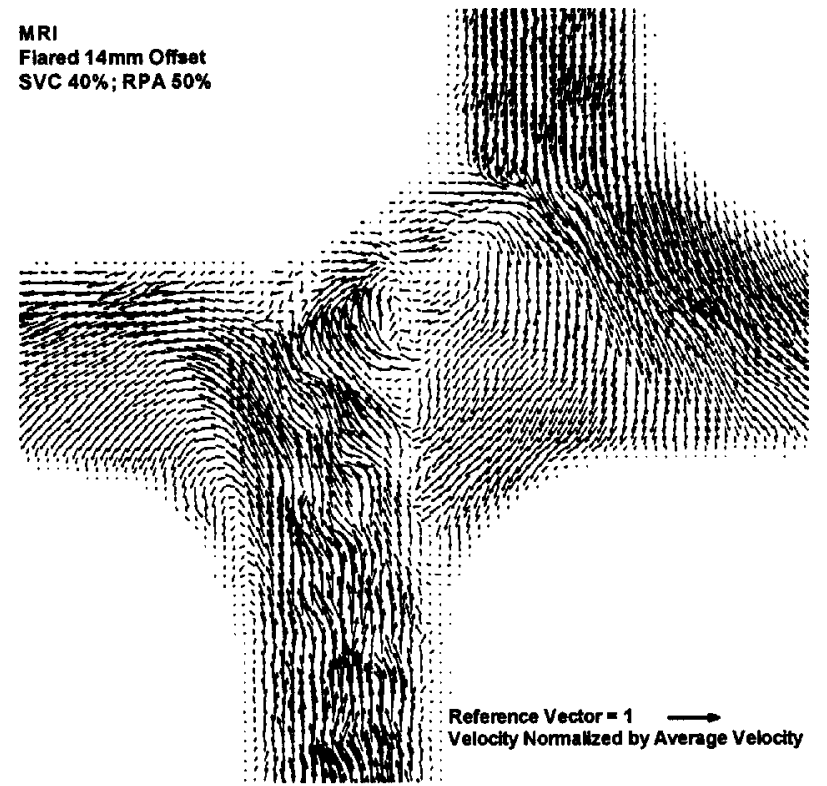

FIGURE 4. Magnetic resonance phase velocity mapping vector plot of flow in the flared $14 \mathrm{~mm}$ (1 caval diameter) offset TCPC model. The SVC:IVC flow ratio was $40: 60$ with $50 \%$ of the total flow to the RPA.

create a mask of that region, and filter the data using this ROI mask. The region of interest (the connection site) was outlined manually using the magnitude images that clearly show signal contrast at the TCPC connection. Based on the outlined ROI, a mask was created to keep only the velocity information inside the TCPC. The resulting files from Transform were loaded into Tecplot for visualization. Tecplot was also used to perform a two pass smoothing routine using the under-relaxed iterative solution to the point-Jacobi Laplace equation. ${ }^{14}$

\section{Normalization of Velocity Results}

In order to directly compare the velocity field results of the DPIV and MRPVM studies, the results were normalized by the average velocity (flow rate/cross-sectional area) for a total flow rate (or cardiac output) of $4 \mathrm{~L} / \mathrm{min}$ in the DPIV study and the Re-matched equivalent (1.147 $\mathrm{L} / \mathrm{min}$ ) in the MRPVM experiments. Normalization was done because the DPIV and MRPVM experiments were run in Re-matched conditions, in the same glass prototype models, but with two different working fluids (DPIV-aqueous $\mathrm{NaI} /$ glycerin solution with kinematic viscosity $=3.5 \mathrm{cSt}$; MRPVM — water with kinematic viscosity $=1.0 \mathrm{cSt}$ ).

\section{RESULTS}

Vector plots shown in Figs. 3-14 use arrows to represent the magnitude and direction of the velocity mea- 

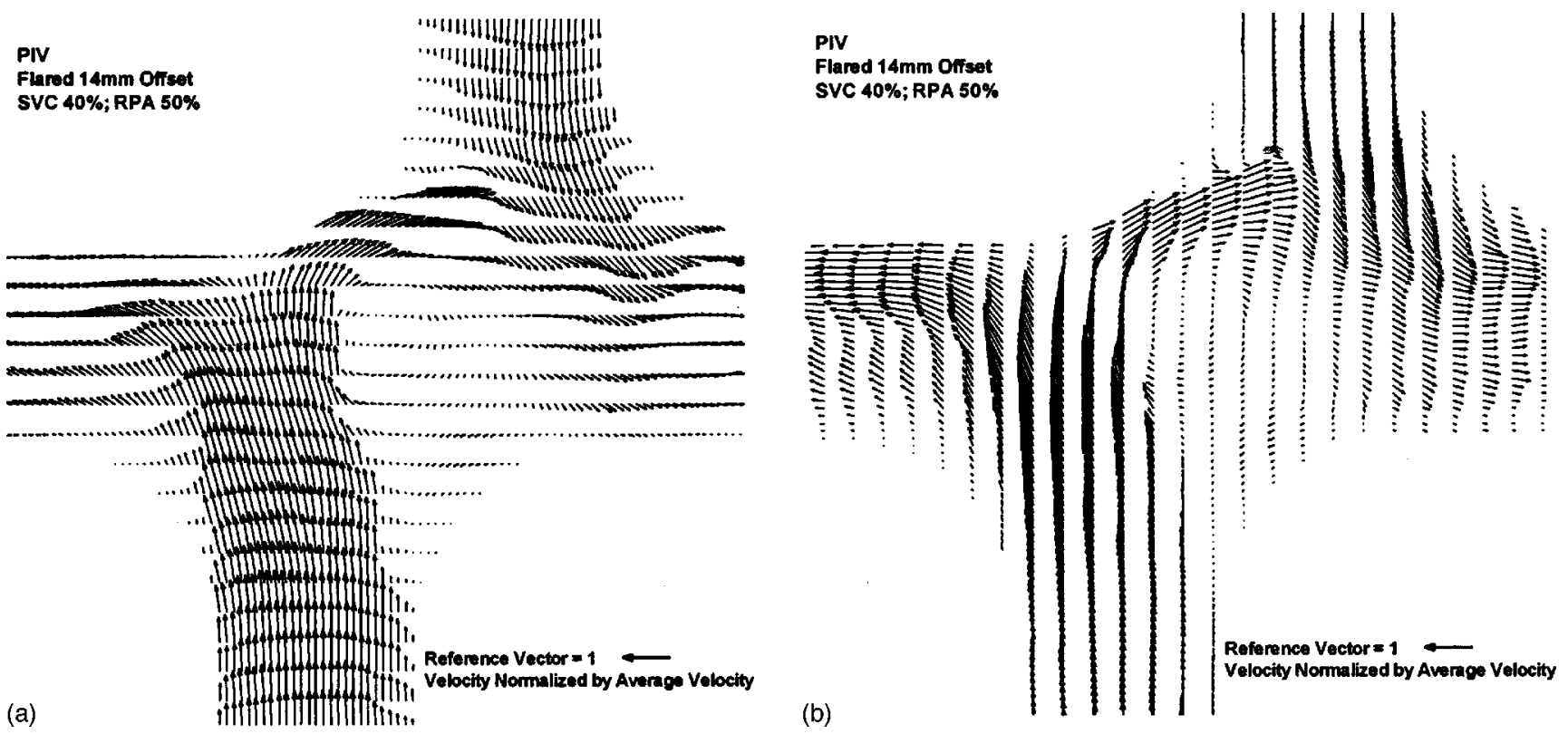

FIGURE 5. Digital particle image velocimetry vector plots of flow in the flared $14 \mathrm{~mm}$ (1 caval diameter) offset TCPC model. The SVC:IVC flow ratio was $\mathbf{4 0 : 6 0}$ with $\mathbf{5 0 \%}$ of the total flow to the RPA. (a) Raw velocity plotted skipping data in the superiorinferior direction. (b) Raw velocity plotted skipping data in the right-left direction.

sured with DPIV and MRPVM. All three spatial components of the velocity vector were acquired in the MRPVM studies, in contrast to the DPIV studies, where only the two in-plane velocity components were available. Therefore, only the in-plane velocity vectors were compared between DPIV and MRPVM in Figs. 3-14.
The results are organized to, first, compare the DPIV and the MRPVM results in one model (flared $14 \mathrm{~mm}$ offset or 1 caval diameter offset) at a constant pulmonary artery flow (50\% to the RPA), and second to compare the results from both techniques over the range of pulmonary flow splits in the straight $21 \mathrm{~mm}$ (1.5 caval diameter)
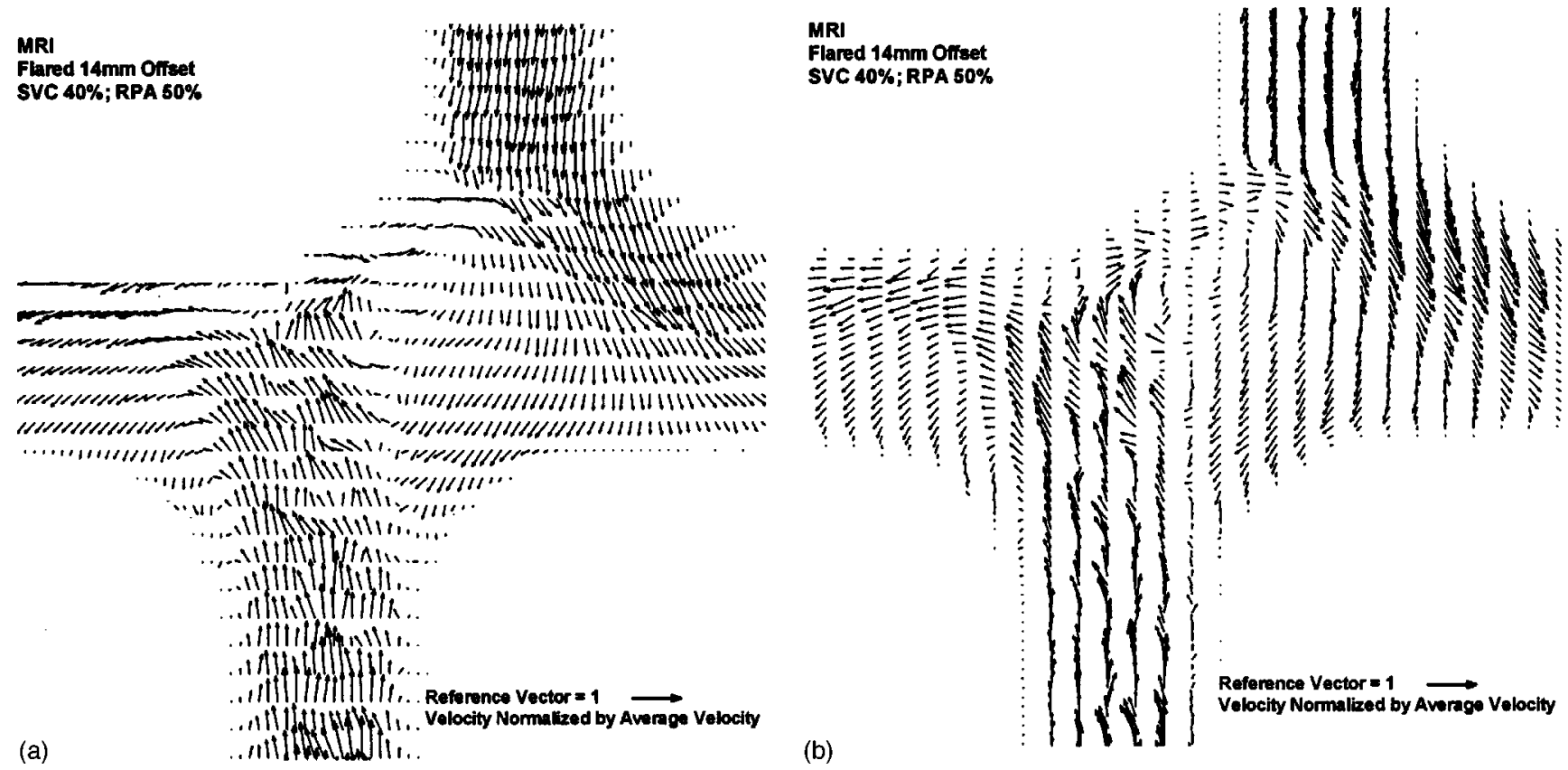

FIGURE 6. Magnetic resonance phase velocity mapping vector plots of flow in the flared $14 \mathrm{~mm}$ (1 caval diameter) offset TCPC model. The SVC:IVC flow ratio was $40: 60$ with $50 \%$ of the total flow to the RPA. (a) Raw velocity plotted skipping data in the superior-inferior direction. (b) Raw velocity plotted skipping data in the right-left direction. 


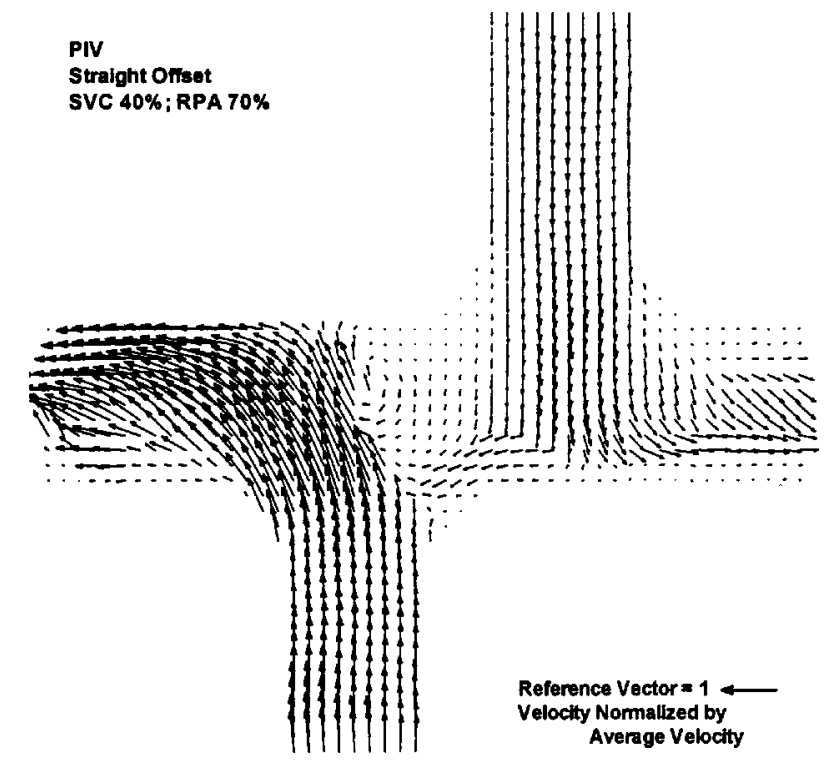

FIGURE 7. Digital particle image velocimetry vector plot of flow in the straight $21 \mathrm{~mm}$ (1.5 caval diameter) offset TCPC model. The SVC:IVC flow ratio was $40: 60$ with $70 \%$ of the total flow to the RPA.

offset and the flared zero offset models. The anatomic directions (superior, inferior, right, and left) are referenced as shown in Fig. 1.

The DPIV normalized velocity in the flared $14 \mathrm{~mm}$ offset model, with equal flow to each pulmonary artery (RPA/LPA 50/50), is plotted in Fig. 3. The MRPVM normalized velocity for the same flow condition is shown

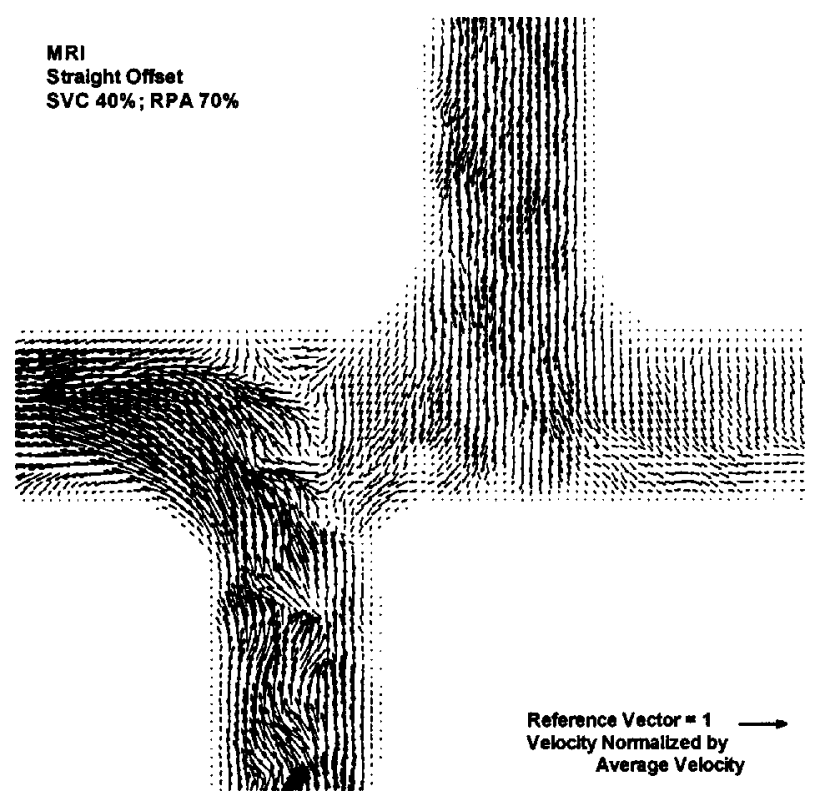

FIGURE 8. Magnetic resonance phase velocity mapping vector plot of flow in the straight $21 \mathrm{~mm}$ (1.5 caval diameter) offset TCPC model. The SVC:IVC flow ratio was 40:60 with $70 \%$ of the total flow to the RPA.

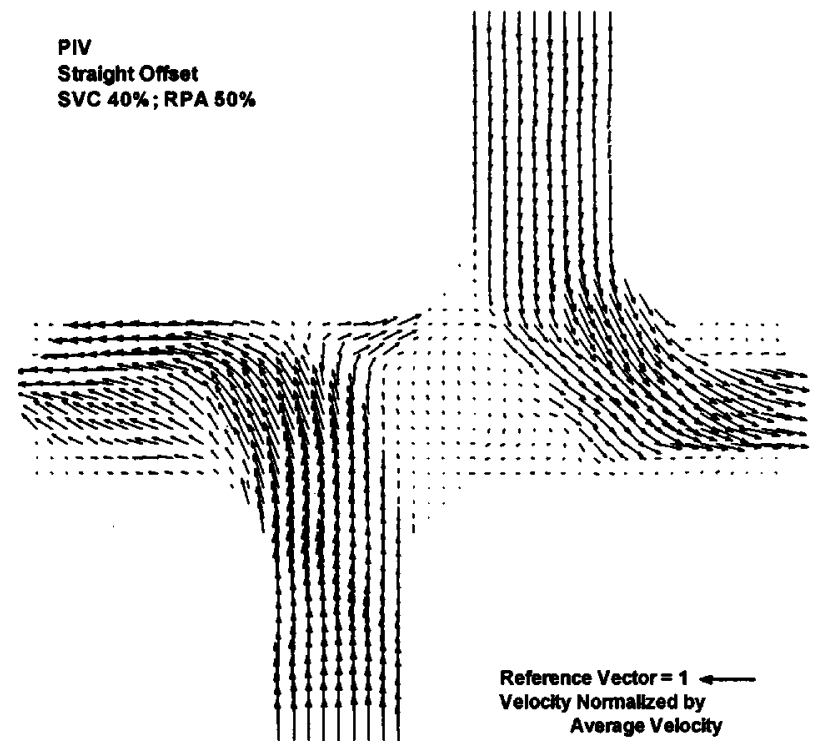

FIGURE 9. Digital particle image velocimetry vector plot of flow in the straight $21 \mathrm{~mm}$ (1.5 caval diameter) offset TCPC model. The SVC:IVC flow ratio was $40: 60$ with $50 \%$ of the total flow to the RPA.

in Fig. 4 for comparison. The comparison of flow fields shows the same general bulk flow features using each technique. The IVC flow ( $60 \%$ of total flow) enters the connection, impacts the superior RPA wall while splitting into two streams, which exit through the RPA and LPA (shown in Figs. 3 and 4). The SVC flow is streamlined and follows the flare of the SVC-to-LPA anasto-

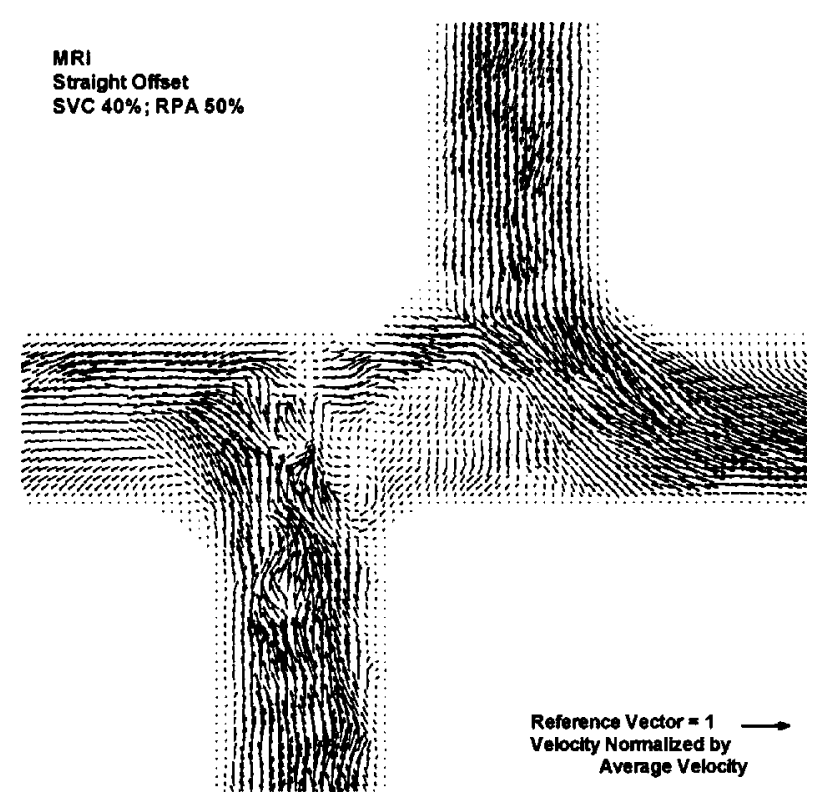

FIGURE 10. Magnetic resonance phase velocity mapping vector plot of flow in the straight $21 \mathrm{~mm}$ (1.5 caval diameter) offset TCPC model. The SVC:IVC flow ratio was 40:60 with $50 \%$ of the total flow to the RPA. 


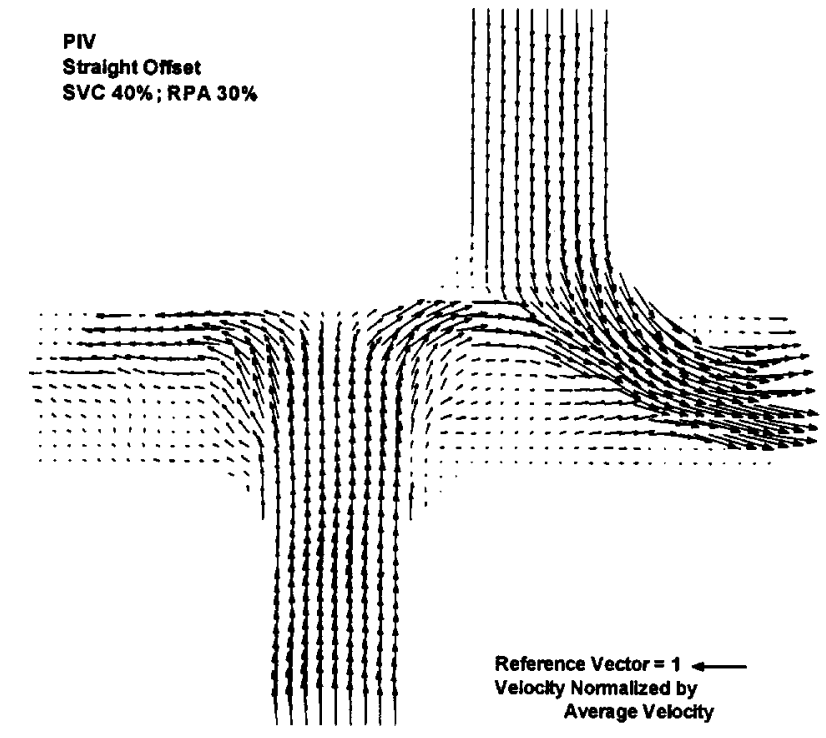

FIGURE 11. Digital particle image velocimetry vector plot of flow in the straight $21 \mathrm{~mm}$ (1.5 caval diameter) offset TCPC model. The SVC:IVC flow ratio was $40: 60$ with $30 \%$ of the total flow to the RPA.

mosis to exit the LPA. The central region between caval inlets is a vortex-like low velocity region, which measured approximately $15 \mathrm{~mm}$ in diameter using both techniques. The values for normalized velocity were also very similar. Comparing the IVC velocity profiles approximately $2.8 \mathrm{~cm}$ inferior to the pulmonary artery centerline, the DPIV measured a peak normalized velocity

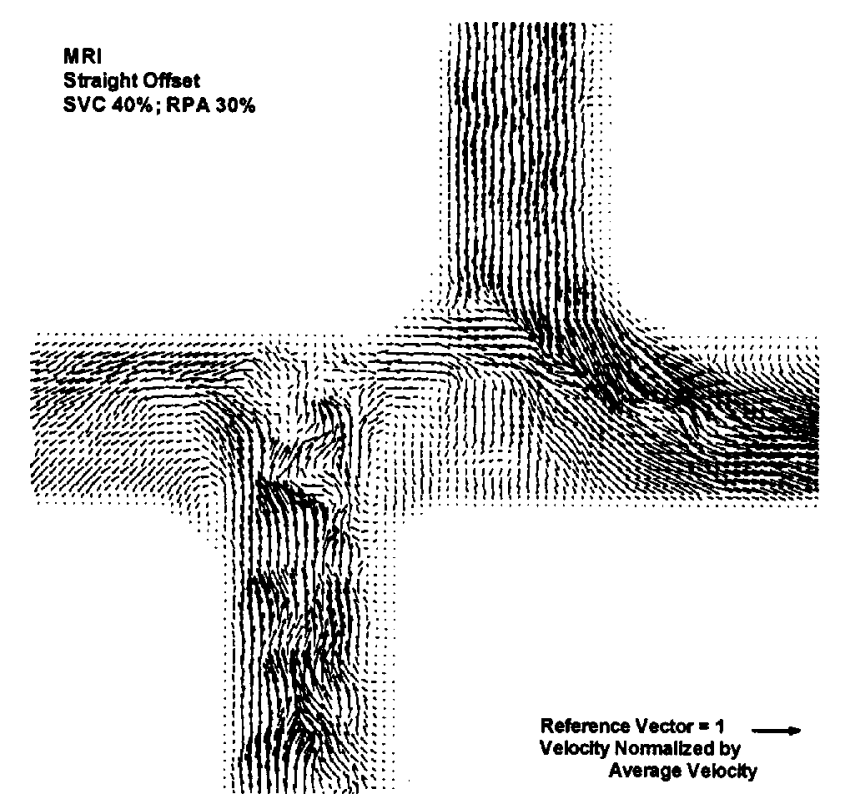

FIGURE 12. Magnetic resonance phase velocity mapping vector plot of flow in the flared $21 \mathrm{~mm}$ (1.5 caval diameter) offset TCPC model. The SVC:IVC flow ratio was 40:60 with $30 \%$ of the total flow to the RPA.

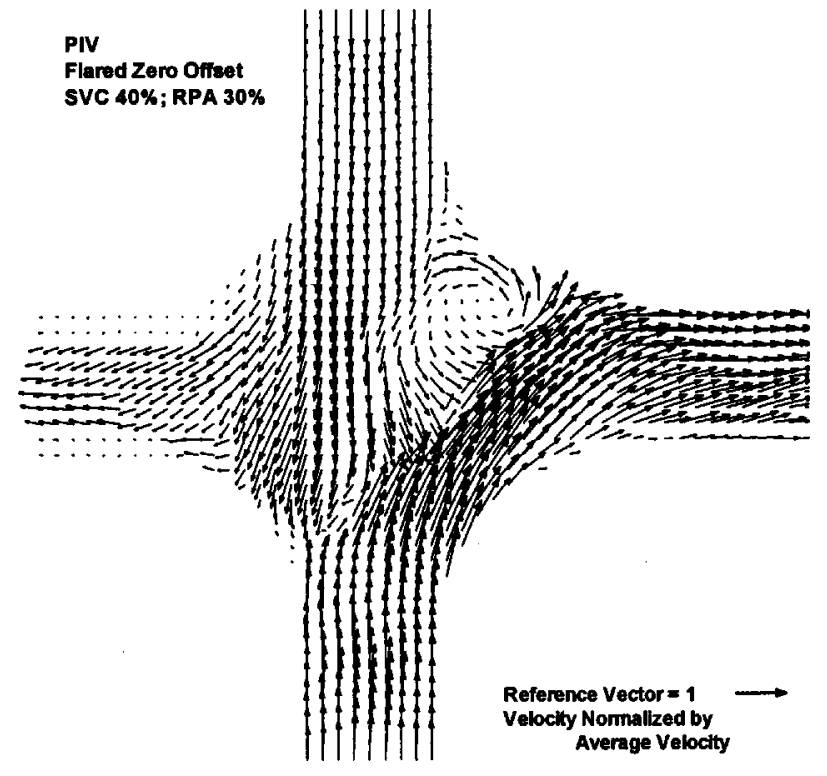

FIGURE 13. Digital particle image velocimetry vector plot of flow in the flared zero offset TCPC model. The SVC:IVC flow ratio was $40: 60$ with $30 \%$ of the total flow to the RPA.

magnitude of 0.89 while the MRPVM result was 0.84 . A similar result was obtained in the SVC approximately 2.8 $\mathrm{cm}$ superior to the pulmonary artery centerline, where the peak normalized velocity magnitude was 0.70 for DPIV and 0.80 for MRPVM. The DPIV data (Fig. 3) appear smoother and show less flow disturbance than the MRPVM data (Fig. 4), where the flow disturbances are

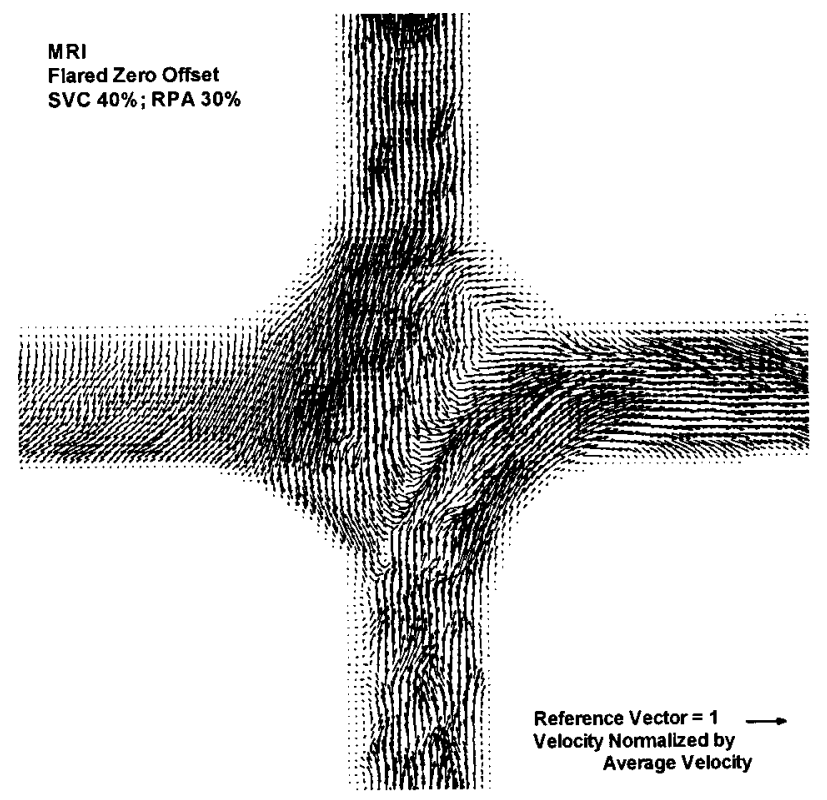

FIGURE 14. Magnetic resonance phase velocity mapping vector plot of flow in the flared zero offset TCPC model. The SVC:IVC flow ratio was $40: 60$ with $30 \%$ of the total flow to the RPA. 
evident, especially in the IVC inlet and in the IVC stream that splits to the LPA. The MRPVM also shows some flow separation at the IVC-to-RPA anastomosis not clearly seen in the DPIV results.

Figures 5(a), 5(b), 6(a), and 6(b) are vector plots of the same dataset shown in Figs. 3 and 4, but the plots were generated by skipping data in the superior-inferior direction [Figs. 5(a) and 6(a)] and in the left-right direction [Figs. 5(b) and 6(b)], to more clearly focus on the velocity and flow patterns measured using each technique. Figure 5 contains plots of the DPIV data and Fig. 6 shows the results of the MRPVM acquisition. In Fig. 5(a), the DPIV inlet profile for the IVC is slightly skewed toward the right. (The anatomic directions in Fig. 1 are used as the reference.) The inlet SVC profile is similar to the IVC but, with more pronounced skewing toward the left. In contrast, Fig. 6(a) shows the IVC inlet velocity profile from the MRPVM data as somewhat disturbed, with no obvious skewing toward either right or left. The SVC inlet in Fig. 6(a) is also skewed slightly toward the left. Although there were differences in the inlet velocity profiles, the normalized velocity magnitudes were in the range of -1.0 to +1.0 using both techniques. Both techniques [Figs. 5(b) and 6(b)], show that the fluid enters the connection from the IVC and is preferentially directed toward the RPA, except very close to the superior wall of the RPA, where a stagnation point is evident. The stagnation point for this model and flow condition occurred approximately $3 \mathrm{~mm}$ to the right of the medial side of the SVC as measured with both techniques. In addition, some of the IVC flow splits toward the LPA. This stream directed toward the LPA is stronger in the DPIV results [Fig. 5(b)] with normalized velocity magnitudes reaching 0.7 in comparison to the MRPVM results [Fig. 6(b)] which measured up to 0.5 . Both techniques show higher velocities along the superior RPA (normalized velocity magnitude ranging $0.7-0.85$ ), with velocities very close to zero at the IVC-to-RPA flare. In the central region between the caval inlets, DPIV (Fig. 5) shows a near zero in-plane velocity, while MRPVM (Fig. 6) indicates a clockwise low velocity region (normalized velocity magnitude $0.0-0.3$ ).

The effect of pulmonary flow split on the flow patterns in the straight $21 \mathrm{~mm}$ offset model is seen in Figs. $7-12$. Figures 7, 9, and 11 are the DPIV results at $70 \%$, $50 \%$, and $30 \%$ of the total flow to the RPA, respectively. Figures 8, 10, and 12 are the MRPVM results for the same flow conditions. Figures 7 and 8 show that, in the straight $21 \mathrm{~mm}$ offset model with $70 \%$ of the flow to the RPA, all of the IVC flow exits to the RPA. A stagnation point occurred on the inferior side of the LPA and was aligned with the center of the SVC inlet using both techniques. The SVC flow which impacted the LPA wall was then forced to split toward both pulmonary arteries. There was minimal interaction between the caval inlets, with a small portion of the SVC flow splitting toward the RPA and the majority exiting the LPA. This was clearly illustrated with both the DPIV (Fig. 7) and MRPVM (Fig. 8). Using DPIV and MRPVM the maximum normalized velocity magnitudes in the IVC stream were 1.1 and 1.0, respectively. Those maximum values were located in the central IVC immediately downstream of the IVC-to-PA anastomosis. Similar values were measured along the superior aspect of the RPA using both techniques. As seen in the flared $14 \mathrm{~mm}$ offset model, again there was some discrepancy in the results from the central region between caval inlets. The DPIV results indicated minimal flow activity, and the MRPVM results showed a low velocity flow moving from the SVC right toward the IVC inlet.

Figures 9 and 10 are results for the same straight 21 $\mathrm{mm}$ offset model at an equally distributed pulmonary flow ratio (50\% of the total flow to the RPA). As the percentage of flow to the LPA increased, all of the SVC flow exited to the LPA. The IVC flow impacted the superior side of the RPA, and distributed flow to both the LPA and RPA. This resulted in a central region of disturbed flow, positioned slightly left of the connection centerline, as well as a small stream of IVC flow joining the SVC flow to exit the LPA. MRPVM and DPIV both agreed with respect to these bulk flow characteristics. A notable difference between the two techniques was the significant IVC disturbance in the MRPVM (Fig. 10), not present in the DPIV results (Fig. 9).

Figures 11 and 12 show the results for the DPIV and MRPVM data, respectively, when $30 \%$ of the total flow exited the RPA. In this situation, the IVC flow $(60 \%$ of the total caval flow) impacts the superior RPA wall and splits to both pulmonary arteries. This resulted in flow separation both in the central region between the cavae, and also along the inferior aspect of the RPA. Also noticeable is the impingement of the IVC flow stream into the SVC, squeezing the SVC flow. This impingement of IVC flow into the SVC caused a local increase in velocity at the SVC-to-LPA junction, captured with both techniques. The MRPVM results indicated a maximum normalized velocity magnitude of 1.0 , while the DPIV result was slightly larger at 1.1 .

One noticeable effect of pulmonary flow split (shown in Figs. 7-12) was the change in the region of low velocity flow between the caval inlets. With $70 \%$ of the total flow exiting the RPA, there was a region of low velocity flow which extended approximately $7 \mathrm{~mm}$ to the right of the SVC along the superior aspect of the pulmonary artery between caval inlets (Figs. 7 and 8). As the percentage of flow to the RPA decreased to $50 \%$ of the total flow, the SVC flow was forced to exit the LPA. Another region of flow separation was formed along the inferior aspect of the pulmonary artery between caval inlets. Bordered on the superior side by the IVC stream 
splitting toward the LPA, the separation region extended approximately $18 \mathrm{~mm}$ to the left of the entering IVC stream (Figs. 9 and 10). At RPA 50\%, the initiation of a second smaller region of low velocity flow also occurred at the IVC-to-RPA junction and extended down the RPA (shown as flow separation in the MRPVM result-Fig. 10). Finally, when the minimum flow exited the RPA $(30 \%$ of the total), regions of flow separation were evident on both the left and right side of the entering IVC stream (Figs. 11 and 12). Between caval inlets, the flow separation extended approximately $21 \mathrm{~mm}$ to the left of the IVC. Entering the RPA, the fluid separation extended out of the field of view and encompassed almost one half of the RPA diameter.

The flared $14 \mathrm{~mm}$ offset (1 caval diameter) and straight $21 \mathrm{~mm}$ offset (1.5 caval diameter) models had minimal caval flow interaction with distinct regions of low velocity flow between caval inlets. In contrast, the flared zero offset model had the maximum caval flow interaction. All flow splits $(70 \%, 50 \%$, and $30 \%$ of the total flow to the RPA) of the flared zero offset model involved significant caval flow interaction. Figures 13 and 14 show the DPIV and MRPVM results, respectively, for the flared zero offset model with $30 \%$ of the total flow to the RPA. This flow condition illustrates the important caval flow interaction, also seen at the other two flow splits. In comparing the results measured with DPIV and MRPVM, again, both techniques illustrate the same large scale flow features. In the flared zero offset model, the caval inlet flows collide. The SVC flow primarily exits the RPA and the IVC flow follows the flare of the vessel toward the LPA. DPIV and MRPVM both show the SVC flow extending into the entrance of the IVC. The majority of the SVC flow impacts the IVC-toRPA wall before exiting the RPA. The remainder of the SVC flow joins the IVC flow to exit the LPA. This influx of SVC flow appears to squeeze the IVC flow along the IVC-to-LPA anastomosis as evidenced by the higher velocities in this region (DPIV normalized velocity magnitude 1.0-1.3; MRPVM 0.9-1.1). A small counterclockwise vortex at the SVC-to-LPA junction, approximately $10 \mathrm{~mm}$ in diameter, is also evident with both techniques. With the exception of that vortex, there were not any other significant regions of flow disturbance.

\section{DISCUSSION}

With the ability to acquire two or three directional velocity data both in vivo (MRPVM) and in vitro (MRPVM and DPIV), this study focused on evaluating the potential of MRPVM in assessing the fluid mechanics in the TCPC and on determining the effect of connection geometry on the TCPC flow field and energetics of the new connection. The flow fields generated as a result of three specific connection geometries were studied and compared using both DPIV and MRPVM.

From the vector plots, it was evident that offsetting the cavae reduced the interaction between the caval inlets. The flared zero offset model had the largest and strongest interaction/collision of caval flow. This interaction decreased significantly in the $14 \mathrm{~mm}$ (1 caval diameter) flared offset and in the $21 \mathrm{~mm}$ (1.5 caval diameter) straight offset models.

The addition of caval flaring at the anastomosis sites allowed the inlet caval flows to make a less restrictive transition to the pulmonary arteries. The fluid flow patterns between the flared $14 \mathrm{~mm}$ offset model (Figs. 3 and 4) and the straight $21 \mathrm{~mm}$ offset model (Figs. 9 and 10) were very similar, with the exception that the region of low velocity flow between caval inlets appeared to have more of a clockwise rotation in the flared $14 \mathrm{~mm}$ offset model.

The importance of caval flow interaction is an area under current investigation. Nevertheless, from an efficiency standpoint, the colliding and intense recirculating flow causes a decrease in forward momentum and potentially less efficient transport of blood to the pulmonary arteries. Because the long term importance of subtle geometric changes (such as those modeled in this study) is unclear, we feel it is important to continue to develop specific empirical relationships between the fluid mechanics of the TCPC and efficiency. From previous studies of power losses as a function of prototype geometry (Sharma et al. and Ensley et al.), the flared zero offset model had the greatest power loss of all three models, over the range of pulmonary flow splits studied (an average of $15.3 \%$ greater than the straight $21 \mathrm{~mm}$ offset and an average of $40.9 \%$ greater than the flared $14 \mathrm{~mm}$ offset model). The caval collision/interaction and resulting vortex structure in the flared zero offset model (Figs. 13 and 14) seen at all flow splits may be an indicator of increased power loss, when compared to the flow fields of the other two models. Pulmonary artery flow split did affect the flow patterns within the TCPC connection, as illustrated in the straight $21 \mathrm{~mm}$ offset model (Figs. 7-12), but a correlation between the flow patterns measured with DPIV and MRPVM and the previously measured power loss was unclear. The flow fields of the flared $14 \mathrm{~mm}$ (1 caval diameter) offset and the straight $21 \mathrm{~mm}$ (1.5 caval diameter) offset models were similar (Figs. 3, 4, 9, and 10), yet the power loss data showed that the straight $21 \mathrm{~mm}$ offset model had an average of $22.5 \%$ greater power losses than the flared $14 \mathrm{~mm}$ offset model, over the range of pulmonary flow splits. This result indicated that the introduction of flaring may be energetically more efficient. In terms of a direct correlation between the DPIV or MRPVM measured fluid mechanics and power loss, the limited information acquired along the symmetry plane of the TCPC model was not 
sufficient to capture the complexity of the fluid structures present and additional data would be needed to make a clear correlation.

This study compared the results from MRPVM and DPIV and found that the bulk flow characteristics specific to a given TCPC prototype geometry are easily evaluated with both techniques and that the normalized velocity magnitudes were very comparable. In addition, when compared to the results from previous qualitative particle flow visualization (PFV) in the same prototype models (Sharma et al. and Ensley et al.), the bulk fluid flow patterns were in good agreement. PFV showed that flow through the prototype connections also had a complex three dimensional motion, especially as flow exits the pulmonary arteries. The 2D DPIV technique used did not capture this complex motion, but in the $4 \mathrm{~mm}$ thick slice, MRPVM measured the normalized through-plane component of velocity to be $\leqslant 30 \%$ of the in-plane components. In the flared $14 \mathrm{~mm}$ offset model, the throughplane component was strongest in the region between caval centers and along the inferior aspect of the RPA. The flared zero offset model had regions of elevated through-plane velocity corresponding with the flow of the SVC and IVC streams as they transitioned to the pulmonary artery, while in the straight $21 \mathrm{~mm}$ offset model the through-plane velocity was greatest in the IVC stream at the level of the connection and also along the inferior aspect of the RPA. Although a comparison of the anterior-posterior velocities was not possible between the techniques, this unique ability of MRPVM to characterize the three dimensionality of the flow field may be an important advantage of using MRPVM in future TCPC evaluation.

In the comparison of DPIV and MRPVM results, we found that DPIV results showed a very smooth flow field, representative of what might be expected from computational studies, in contrast to the MRPVM results, which showed more flow disturbances. A limitation and probable explanation for the flow disturbances seen especially in the inlets of the MRPVM data was a difference in the experimental flow loops. For the DPIV and previously published particle flow visualization studies, the entrance lengths for the superior and inferior vena cava were substantially longer than those used in the MRPVM experiments. Due to limited space constraints in the MRI scanner, the entrance lengths were shorter, causing these entrance effects to be magnified in the MRPVM result. The flow loop contained a $90^{\circ}$ elbow plus connections to the model approximately $14 \mathrm{~cm}$ upstream of the caval inlets. Differences in the inlet velocity profiles measured with both techniques are attributed to these entrance flow effects.

There are also three additional possible explanations for the differences seen between the DPIV and MRPVM. First was the differences in slice thickness and in-plane resolution between the two experimental protocols. DPIV measured the velocity within a very thin plane of the model $(\leqslant 1 \mathrm{~mm})$, but the MRPVM data was an average of velocities through a $4 \mathrm{~mm}$ thick slice of data. This larger volume for the MRPVM data points could contribute to the result of small velocities measured in the central regions between cavae with MRPVM, while minimal or near zero velocity flow was measured using DPIV. A second consideration is the differences in inplane resolution between the techniques. Using DPIV, the sensitivity of the displacement/time or velocity measurement is a function of the camera pixel resolution and interrogation region dimension. In this study, the camera resolution was $1008 \times 1018$ pixels over a field of view of $6.6 \times 6.8 \mathrm{~cm}^{2}$. Since the interrogation region was chosen to be $64 \times 64$ pixels, a cross-correlation result was produced every $0.4 \times 0.4 \mathrm{~cm}^{2}$. This resolution may not be sensitive to small spatial variations in the in-plane velocity, causing the flow field results to appear very smooth. Finally, the DPIV data presented was an average of 16 instantaneous velocity field measurements, averaged over a time of approximately $1.6 \mathrm{~s}$. This time scale is significantly different from the MRPVM acquisition time, which was a combination of single velocity encoded acquisitions with a combined scan time of approximately $2.3 \mathrm{~min}$ for two components of velocity.

There were several limitations to the study and to the techniques used for velocity field measurement. Already mentioned were the differences in experimental flow loops due to the space constraints of the clinical MR scanner. Also, this study was conducted under steady flow conditions which differ from the in vivo situation where flow through the TCPC has been reported to have a phasic nature. Because the pulsatility of flow in this region is relatively small, the introduction of pulsatile flow is not expected to significantly change the results of the comparison between techniques, although different data acquisition protocols would be necessary. Caution should be taken when focusing on the near wall velocities measured with both DPIV and MRPVM. In each case, determination of the vessel walls was performed manually, without any subpixel vessel wall determination, leading to possibly significant partial volume effects at the vessel walls. Finally, the comparison of the velocity flow field was limited to the symmetry plane of the prototype TCPC models. This plane was chosen for comparison in order to correctly align the two data sets, and to minimize the partial volume effects which would be exaggerated even further in off-center planes, especially in the MRPVM measurements which had a $4 \mathrm{~mm}$ slice thickness. 


\section{CONCLUSIONS}

This study found that the large scale flow fields measured with MRPVM were in agreement with the DPIV result. The location of stagnation points, the size of flow separation regions and location of vortex formations, as well as the normalized velocity magnitudes in important areas of the connection geometries were in agreement between the two techniques. In addition, the ability of MRPVM to measure the third, through-plane component of velocity may be important in future TCPC connection evaluation and in reconstruction of the full flow field.

The absence of caval offset was found to cause significant caval flow collision at the connection site. Offsetting the caval inlets (flared $14 \mathrm{~mm}$ or 1 caval diameter offset and straight $21 \mathrm{~mm}$ or 1.5 diameter caval offset) reduced the caval flow interactions and caused fluid separation regions between the caval inlets and in the pulmonary artery as well. The direct caval flow collision in the zero offset model correlated with the greatest power loss previously measured in the three models.

Comparison of MRPVM results with those from DPIV indicate that MRPVM can be used to evaluate the velocity flow fields of prototype TCPC models in vitro and in turn has the potential to be a useful clinical tool for noninvasive in vivo TCPC physiologic/functional evaluation.

\section{ACKNOWLEDGMENTS}

The authors acknowledge the skillful work of Jerry A. Cloninger in constructing the glass models for this study. This work was supported by grants from the National Institutes of Health (HL-52799) and the American Heart Association, Georgia Affiliate.

\section{REFERENCES}

\footnotetext{
${ }^{1}$ Bluestein, D., L. Niu, R. Schoephoerster, and M. Dewanjee. Steady flow in an aneurysm model: Correlation between fluid dynamics and blood platelet deposition. J. Biomech. Eng. 118:280-286, 1996.

${ }^{2}$ Bryant, D., J. Payne, D. Firmin, and D. Longmore. Measurement of flow with NMR imaging using a gradient pulse and phase difference technique. J. Comput. Assist. Tomogr. 8:588-593, 1984.

${ }^{3}$ Chatzimavroudis, G., P. Walker, J. Oshinski, R. Franch, R. Pettigrew, and A. Yoganathan. The importance of slice location on the accuracy of aortic regurgitation measurements with magnetic resonance phase velocity mapping. Ann. Biomed. Eng. 25:644-652, 1997.
}

${ }^{4}$ Ensley, A., P. Lynch, G. Chatzimavroudis, C. Lucas, S. Sharma, and A. Yoganathan. Toward designing the optimal total cavopulmonary connection: An in vitro study. Ann. Thorac. Surg. 68:1384-1390, 1999

${ }^{5}$ Firmin, D., G. Nayler, R. Klipstein, S. Underwood, R. Rees, and $\mathrm{D}$. Longmore. In vivo validation of MR velocity imaging. J. Comput. Assist. Tomogr. 11:751-756, 1987.

${ }^{6}$ Fontan, F., and E. Baudet. Surgical repair of tricuspid atresia. Thorax 26:240-248, 1971.

${ }^{7}$ Karn, E., S. Beale, A. Duitino, T. Wei, A. Graham, and G. Nackman. Development of wall surface tangent DPIV measurement techniques for arterial branch models. J. Biomech. Eng. 120:784-787, 1998.

${ }^{8}$ Kim, Y. H., P. G. Walker, A. A. Fontaine, S. Panchal, A. E. Ensley, J. Oshinski, S. Sharma, B. Ha, C. L. Lucas, and A. P. Yoganathan. Hemodynamics of the Fontan connection: An in-vitro study. J. Biomech. Eng. 117:423-428, 1995.

${ }^{9}$ Laks, H., A. Ardehali, P. W. Grant, L. Permut, A. Aharon, M. Kuhn, J. Isabel-Jones, and A. Galindo. Modification of the Fontan procedure: Superior vena cava to left pulmonary artery connection and inferior vena cava to right pulmonary artery connection with adjustable atrial septal defect. Circulation 91:2943-2947, 1995.

${ }^{10}$ de Leval, M. R., P. Kilner, M. Gewillig, and C. Bull. Total cavopulmonary connection: A logical alternative to atriopulmonary connection for complex Fontan operations. J. Thorac. Cardiovasc. Surg. 96:682-695, 1988.

${ }^{11}$ Lim, W., Y. Chew, T. Chew, and H. Low. Steady flow velocity field and turbulent stress mappings downstream of a porcine bioprosthetic aortic valve in vitro. Ann. Biomed. Eng. 25:86-95, 1997.

${ }^{12}$ Lins, R., M. Lins, C. Cavalcanti, R. Miranda, and J. Mota. Orthoterminal correction of congenital heart disease: Double cava-pulmonary anastomosis. J. Thorac. Cardiovasc. Surg. 84:633-635, 1982.

${ }^{13}$ Meier, D., S. Maier, and P. Bosiger. Quantitative flow measurements on phantoms and on blood vessels with MR. Magn. Reson. Rev. 8:25-34, 1988.

${ }^{14}$ Mitchell, A., and D. Griffiths. The Finite Difference Method in Partial Differential Equations. New York: Wiley, 1980.

${ }^{15}$ Mussivand, T., K. Day, and B. Naber. Fluid dynamic optimization of a ventricular assist device using particle image velocimetry. ASAIO J. 45:25-31, 1999.

${ }^{16}$ Raffel, M., C. E. Willert, and J. Kompenhans. Particle Image Velocimetry. New York: Springer, 1998.

${ }^{17}$ Sharma, S., S. Goudy, P. Walker, S. Panchal, A. Ensley, K. Kanter, V. Tam, D. Fyfe, and A. Yoganathan. In vitro flow experiments for determination of optimal geometry of total cavopulmonary connection for surgical repair of children with functional single ventricle. J. Am. Coll. Cardiol. 27:1264-1269, 1996.

${ }^{18}$ Sloth, E., K. Houlind, S. Oyre, W. Kim, E. Pedersen, H. Jorgensen, and J. Hasenkam. Three-dimensional visualization of velocity profiles in the human main pulmonary artery with magnetic resonance phase velocity mapping. Am. Heart J. 128:1130-1138, 1994.

${ }^{19}$ Willert, C., and M. Gharib. Digital particle image velocimetry. Exp. Fluids 10:181-193, 1991. 